\title{
The improvement of the best practice guidelines for preimplantation genetic diagnosis of cystic fibrosis: toward an international consensus
}

\author{
Anne Girardet ${ }^{1,2,3}$, Victoria Viart ${ }^{1,3}$, Stéphanie Plaza ${ }^{1,3}$, Gemma Daina ${ }^{4}$, Martine De Rycke ${ }^{5}$, \\ Marie Des Georges ${ }^{1,3}$, Francesco Fiorentino ${ }^{6}$, Gary Harton ${ }^{7}$, Aliya Ishmukhametova ${ }^{1,3}$, Joaquima Navarro ${ }^{4}$, \\ Caroline Raynal ${ }^{1,3}$, Pamela Renwick ${ }^{8}$, Florielle Saguet ${ }^{1,3}$, Martin Schwarz ${ }^{9}$, Sioban SenGupta ${ }^{10}$, Maria Tzetis ${ }^{11}$, \\ Anne-Françoise Roux ${ }^{1,3}$ and Mireille Claustres ${ }^{\star, 1,2,3}$
}

Cystic fibrosis (CF) is one of the most common indications for preimplantation genetic diagnosis (PGD) for single gene disorders, giving couples the opportunity to conceive unaffected children without having to consider termination of pregnancy. However, there are no available standardized protocols, so that each center has to develop its own diagnostic strategies and procedures. Furthermore, reproductive decisions are complicated by the diversity of disease-causing variants in the CFTR (cystic fibrosis transmembrane conductance regulator) gene and the complexity of correlations between genotypes and associated phenotypes, so that attitudes and practices toward the risks for future offspring can vary greatly between countries. On behalf of the EuroGentest Network, eighteen experts in PGD and/or molecular diagnosis of CF from seven countries attended a workshop held in Montpellier, France, on 14 December 2011. Building on the best practice guidelines for amplification-based PGD established by ESHRE (European Society of Human Reproduction and Embryology), the goal of this meeting was to formulate specific guidelines for CF-PGD in order to contribute to a better harmonization of practices across Europe. Different topics were covered including variant nomenclature, inclusion criteria, genetic counseling, PGD strategy and reporting of results. The recommendations are summarized here, and updated information on the clinical significance of CFTR variants and associated phenotypes is presented.

European Journal of Human Genetics (2016) 24, 469-478; doi:10.1038/ejhg.2015.99; published online 27 May 2015

\section{INTRODUCTION}

Cystic fibrosis (CF, OMIM \#219700) is one of the most common life-shortening autosomal recessive disorders in populations of European descent, affecting about 1 in 4000 individuals. ${ }^{1}$ It is also present in most other ethnicities, but the disease frequency varies greatly. $\mathrm{CF}$ is caused by sequence variations in the Cystic fibrosis transmembrane conductance regulator (CFTR, OMIM \#602421) gene on $7 \mathrm{q} 31.2$, which lead to absent or decreased chloride and bicarbonate transports across the apical membranes of secretory epithelial cells, elevated intracellular sodium and decreased extracellular water. This results in thickened secretions in affected structures (airways, pancreatic and biliary ducts, intestines and vas deferens). Although the severity of symptoms is variable, most patients with CF typically present progressive obstructive lung disease with persistent airway colonization and bronchiectasis, pancreatic insufficiency, gastrointestinal and nutritional anomalies, abnormally high sweat chloride levels and male infertility due to absent or occluded vasa deferentia. Early detection of CF through newborn screening programs is of major importance for the improvement of both survival and quality of life of patients. ${ }^{2-4}$
Close to 2000 unique variations have been identified so far in the CFTR gene (http://www.genet.sickkids.on.ca), either in CF, in CFTRrelated disorders (CFTR-RD), or in asymptomatic individuals. The term CFTR-RD refers to a distinct spectrum of nonlethal diseases that do not fulfill diagnostic criteria for CF but show evidence of CFTR dysfunction, ${ }^{5}$ including congenital bilateral absence of vas deferens (CBAVD), ${ }^{6-12}$ recurrent pancreatitis, ${ }^{13,14}$ disseminated bronchiectasis, ${ }^{15}$ or CFTR-related metabolic syndrome ${ }^{16}$ (Box 1, Table 1). Besides the most common CF disease-causing variant, a 3-bp deletion c.1521_1523delCTT known as p.Phe508del, that accounts for approximately $67 \%$ of CFTR mutant alleles in Europe, another 130 variants have been clinically, genetically and experimentally classified as CF-causing. ${ }^{17}$ The clinical consequences of many other variants, particularly those that change an amino acid in the protein (missense variants) are largely uncharacterized. In addition, some pathogenic variants are not necessarily fully penetrant ${ }^{18}$ (eg their deleterious effect depends on other factors). The number of variants of unknown (clinical) significance (VUCS or VUS) is predicted to increase with the expansion of next generation sequencing methods for CF carrier screening, which is among the first general population

\footnotetext{
${ }^{1}$ Laboratoire de Génétique Moléculaire, CHU Arnaud de Villeneuve, Montpellier, France; ${ }^{2}$ Faculté de Médecine, Université Montpellier I, Montpellier, France; ${ }^{3}$ Unité INSERM U827, Montpellier, France; ${ }^{4}$ Unitat de Biologia Cellular i Genetica Medica, Universitat Autonoma de Barcelona, Bellaterra, Spain; ${ }^{5}$ Center for Medical Genetics UZ Brussel, Brussels, Belgium; ${ }^{6}$ Genoma Laboratories, Via di Castel Giubileo, Roma, Italy; ${ }^{7}$ Reprogenetics, Livingston, NJ, USA; ${ }^{8}$ Center for Preimplantation Genetic Diagnosis, Guy's and St Thomas' NHS Foundation Trust, Great Maze Pond, London, UK; ${ }^{9}$ Manchester Centre for Genomic Medicine, Manchester, UK; ${ }^{10}$ UCL Center for PGD, Institute for Women's Health, University College London, London, UK; ${ }^{11}$ Department of Medical Genetics, University of Athens, Greece

${ }^{*}$ Correspondence: Professor M Claustres, Genetic Diagnosis Laboratory, IURC, 641 avenue du Doyen Gaston Giraud, 34095 Montpellier Cedex 3, 34095, France. Tel: +33 4117598 47; Fax: +33 4117598 82; E-mail: Mireille.Claustres@inserm.fr

Received 10 February 2015; revised 15 April 2015; accepted 17 April 2015; published online 27 May 2015
} 


\section{Box 1 The spectrum of CFTR-associated diseases}

\section{$\checkmark$ Classical or typical cystic fibrosis (CF) includes the following features:}

- Chronic sinopulmonary disease with persistent colonization with typical CF pathogens,

- Specific gastrointestinal or nutritional abnormalities,

- Salt-loss syndromes,

- Obstructive azoospermia,

- Abnormal sweat test (chloride concentration $\geq 60 \mathrm{mmol} / \mathrm{l}$ ),

- Two CF-causing variants in trans.

Over $85 \%$ of these patients have two severe CF-causing variants and are pancreatic insufficient (CF-PI). A minority of CF patients carry at least one 'moderate' variant, which confers some residual CFTR channel function maintaining sufficient exocrine pancreatic function to allow normal digestion of nutrients (pancreatic sufficiency, CFPS).

In many countries, CF is now diagnosed through newborn screening detecting abnormal values of immunoreactive trypsinogen in the blood, on the basis of abnormal sweat test and two CF-causing variants.

$\checkmark$ Nonclassical or atypical CF

A small proportion of patients have symptoms characteristic of CF in at least one organ but a negative $(<30 \mathrm{mmol} / \mathrm{l})$ or borderline $(30-60 \mathrm{mmol} / \mathrm{l}) \mathrm{sweat}$ test. They are CF-PS and exhibit a milder and late lung disease. The diagnosis is confirmed by the identification of variants (either one mild in trans with a severe one, or two mild variants), and by abnormal nasal potential difference measurement or intestinal current measurement using rectal biopsy. Individuals in this group have an increased risk of pancreatitis.

$\checkmark$ CFTR-related disorders (CFTR-RD) ${ }^{5}$

A CFTR-RD is defined as a clinical entity with evident CFTR dysfunction that does not fulfill the CF diagnostic criteria. Three distinct subgroups must be distinguished: congenital bilateral absence of the vas deferens, acute recurrent or chronic pancreatitis, and disseminated bronchiectasis.

Congenital bilateral absence of the vas deferens $(C B A V D)^{6-12}$

CBAVD is the most common cause of infertility in otherwise healthy males, with an incidence of 1:1000 men, and is present in all ethnicities. The male reproductive tract is the most sensitive tissue to CFTR dysfunction, so that CFTR variations not severe enough to cause CF can be responsible for vas deferens aplasia. Up to $80 \%$ of CBAVD men have two CFTR variants in compound heterozygosity, whereas $20 \%$ have either only one or no variant. None of CBAVD men carry two severe CFTR variants: the vast majority carry a mild in trans with a severe variant, and a minor proportion has two mild variants. In CBAVD men from European descent, the two most common genotypes are p.Phe508del in trans either with the '5T allele' in intron 8 (now designated as c.1210-12T[5] in intron 9) (30\%) or with the variants R117H-7T (p. Arg117His associated with a 7T allele (c.1210-12T[7]) (6\%). In humans, the variant 5T, also present in the general population at a frequency of 0.05, alters the splicing signals and leads to the skipping of exon 9 (10 in the HGVS nomenclature) in a proportion of transcripts. The skipping varies among tissues and individuals, which generates a proportion of incomplete and nonfunctional CFTR proteins; the result is a reduced quantity of normal CFTR protein. The disease penetrance of $5 T$ is incomplete (some men carrying a 5T in trans with a severe variant are fertile), and is influenced by another polymorphic sequence immediately upstream, the 'TG tract': the longer the TGn and the shorter the Tn repeats are, the greater is the reduction in full-length CFTR transcripts. CBAVD is more likely to occur in males carrying a $5 T$ associated in cis with 12TG (c.[1210-34TG[12];1210-12T[5]] or 13TG (c.[1210-34TG[13];1210-12T[5]] than 11TG (c.[1210-34TG[11];1210-12T[5]] repeats. Thus the determination of TG repeat number is predictive of the pathogenicity of the $5 T$ alleles.

Spermatogenesis in CBAVD males is normal, thus patients can be treated by sperm extraction followed by ICSI. If the female partner also carries a CF-causing variant, the offspring has an increased risk (25\%) of CF (the CF variants of both partners), and male offspring have, depending on the penetrance of mild variants, up to $25 \%$ additional chance of having CBAVD (the mild variant of the father and the CF variant of the mother). Genetic counselling may be particularly difficult in couples with a CBAVD partner as they often carry variants with a wide range of effects from normal to CFTR-RD. ${ }^{11,12}$

Idiopathic chronic pancreatitis and recurrent acute pancreatitis: approximately $30 \%$ of individuals in this group who do not fulfill the diagnostic criteria for CF are found with at least one CFTR variant and sweat chloride values in the intermediate range. ${ }^{13}$ They include a small proportion with compound heterozygosity for a CF-causing and a mild CFTR variant (including the 5T allele) and a majority of carriers of a single CF or CFTR-RD variant with increased susceptibility to pancreas dysfunction. Recent studies suggest that certain missense variants could selectively alter the bicarbonate transport while chloride function is preserved, thus affecting only tissues that necessitate CFTR for bicarbonate secretion such as pancreas. ${ }^{14}$

Disseminated bronchiectasis: 15-25\% of individuals with abnormal and irreversible dilatation of thick-walled bronchi are found to carry CFTR variants, mostly with residual CFTR function (including a high proportion of allele $5 \mathrm{~T}$ ) and mostly in the heterozygous state. CFTR variants may act as risk alleles for multigenic disorders in the general population. ${ }^{15}$

CFTR-related metabolic syndrome (CRMS): Asymptomatic newborns with an inconclusive diagnosis following a positive NBS test (abnormal immunoreactive trypsinogen levels but nondiagnostic sweat chloride or genetic analysis). They may either remain symptom free or develop mild CF or CFTR-RD later in life, and therefore need regular follow-up. ${ }^{16}$

genetic screening tests, with worldwide probably more than 10 millions tests each year. Assessing disease causality for CFTR variants is extremely challenging ${ }^{18-20}$ owing to their number and variety ${ }^{21}$ and the complexity of genotype-phenotype relationship in CF or CFTRRDs (some identical genotypes may be associated with different phenotypes). Interpreting CFTR variations as 'pathogenic' or 'neutral' and, beyond, as 'CF-causing' or 'non CF-causing', has evident impact on diagnosis, genetic counseling and reproductive choices of patients and families.
Evidence from practice indicates that whilst natural conception followed by prenatal diagnostic (PND) was in the past the most common reproductive choice for many couples at risk of passing on $\mathrm{CF}$, preimplantation genetic diagnosis (PGD) is increasingly being seen as a preferred choice. So far, the most used approach for PGD for CF involves the biopsy of one blastomere from 3 days-old embryos following ICSI (intracytoplasmic sperm injection) at the 6-10 cell stage. 22 Blastomeres are then analyzed and embryos shown to be free of the disease are transferred to the maternal uterus. This procedure 
avoids the dilemma of therapeutic abortion following PND by chorionic villus sampling or amniocentesis in the first or second trimester of pregnancy. As the blastomere biopsy procedure on day 3 can be traumatic to the embryo, reducing its developmental potential, there is an increasing tendency to move to the day 5 or 6 trophectoderm biopsy at the blastocyst stage. ${ }^{23}$ However, blastocyst biopsy is more challenging than blastomere biopsy, and only some centers (especially those that test PGD embryos for aneuploidy ${ }^{24}$ ) have changed their protocols so far. CF is one of the most common indications of PGD worldwide, accounting for almost $10 \%$ of all indications for monogenic disorders, as recorded in the European Society of Human Reproduction and Embryology (ESHRE) PGD consortium data collection XII. ${ }^{25}$

Best practice guidelines for amplification-based PGD dealing with infrastructures, equipment and materials as well as molecular procedures (pre-examination, examination and post-examinations processes) have been established and updated by the ESHRE consortium. ${ }^{26,27}$ These guidelines describe general recommendations on genetic practices for PGD programs, in order to build a consensus opinion on best practices within the PGD community and to achieve a minimum standard across all centers. However, due to the absence of a universal, standardized PGD assay, each center develops its own inhouse methods. Such methods are based on direct screening of specific variants, ${ }^{28,29}$ linkage analysis using informative microsatellites, ${ }^{30,31}$ or a combination of direct and indirect testing. ${ }^{32,33}$ Some centers use fluorescent multiplex PCR protocols, others perform nested PCRs, and a few centers use Whole Genome Amplification techniques (mainly Multiple Displacement Amplification, MDA) to generate larger quantities of DNA template, followed by haplotyping of several polymorphic markers. ${ }^{34,35}$

Many health practitioners, even among highly trained specialists in academic medical institutions and including those in reproductive medicine, wish more information about genetic testing and counseling. ${ }^{36}$ The guidelines presented here are the result of a meeting held in December 2011 with the partnership of EuroGentest (www. eurogentest.org). The main purpose of the meeting was to formulate more specific PGD guidelines for CF-PGD, in line with the general guidelines for amplification-based $\mathrm{PGD},{ }^{27}$ for organization of a PGD center for $\mathrm{PGD} / \mathrm{PGS}^{37}$ and for polar body and embryo biopsy ${ }^{38}$ previously published by ESHRE. The specific guidelines are based on experience with CFTR genetic testing, and will contribute to the harmonization and quality of procedures, methods and protocols and to the standardization of reporting results for cystic fibrosis. We also present updated information on CFTR variation including nomenclature, classification of variants, and genotype-phenotype correlations.

\section{MATERIALS AND METHODS}

In order to discuss relevant technical and genetic issues in PGD for cystic fibrosis, a 'Best practice guidelines for CF-PGD meeting' was held in Montpellier, France, on 14 December 2011. The meeting brought together eighteen experts in the fields of PGD and molecular diagnosis of CF and CFTRRDs from seven countries (Spain, Belgium, Italy, USA, UK, Greece and France). Presentations and subsequent discussions formed the basis for establishing specific guidelines.

\section{NOMENCLATURE}

Since the discovery of the CFTR gene in 1989, the CF scientific community developed its own nomenclature system (Cystic Fibrosis Mutation Database, (http://www.genet.sickkids.on.ca/)). Despite the introduction of a worldwide standardized Human Genome Variation
Society (HGVS) nomenclature, ${ }^{39,40}$ the original ('legacy') names are still commonly used for CFTR variants and polymorphisms in clinical services, whereas diagnostic laboratories use both nomenclatures simultaneously to ensure compatibility with previous clinical reports and understanding by clinicians and couples. The HGVS DNA variant numbering system is based on cDNA, defining position ' +1 ' as the A of the ATG translation initiation codon in the CFTR reference sequence (NM_000492.3), with initiation codon as codon 1 and exons numbered 1-27. In contrast, in the legacy (traditional) annotation, ' +1 ' was the first base of the mRNA major transcript, and the A of the ATG was consequently nucleotide 133. These two different nomenclatures can lead to confusion and serious erroneous interpretation and reporting of the location of variants. For example, the well-known 1717-1G $>A$ and $3120+1 G>A$ variants (legacy names) are designated as c.1585-1G $>$ A and c.2988+1G $>$ A, respectively, according to the HGVS nomenclature. Not being aware of nomenclature changes and testing a variant at a wrong position in the gene will ultimately lead to erroneous PND or PGD. Variants nomenclature is a complex and evolving process, therefore constant vigilance is needed for accurate reporting. ${ }^{41}$

It is strongly recommended to obtain the original molecular genetic reports including the description of identified variants in both the HGVS and legacy nomenclatures. Then, it is recommended to verify the reported variants on new DNA samples from the couples, using standard molecular techniques (Box 2).

Traditional and HGVS nomenclatures for the most common CF-causing, non CF-causing and variants with varying clinical consequences, and examples of common CFTR genotypes nomenclatures, are presented in Tables 1 and 2, respectively. Useful Internet resources are compiled in Box 3.

\section{INCLUSION CRITERIA}

Multiple occasions arise to detect individuals carrying CFTR variants (Table 3), who may become future candidates for PGD. Some variants are not causing CF but have been included in commercially available population-based carrier screening panels. These kits may be used by accredited and non-accredited laboratories, thus accreditation does not guarantee the non-detection of clinically non-significant variants. Couples may not have been correctly counseled about the risks. ${ }^{42,43}$ Before enrolling a couple for a PGD procedure for $\mathrm{CF}$, it is recommended to evaluate within a multidisciplinary team whether the variants identified in both partners should be considered for PND or PGD. Variants may be grouped in four categories: (i) loss-of-

Box 2 Importance of verifying the presence of variants initially reported in each member of the couple requesting a CF-PGD

Analysing the DNA of each member of the couple will confirm the initial result and will confirm that both parents carry at least one CF-causing variant.

For example, inability to confirm the variants in both parents may be found in cases with apparent homozygosity for a common or a rare variant:

- Nonpaternity

- Presence on the opposite allele of a large deletion, nonrevealed by routine tests

- Presence of a rare SNP in primer-binding sites or a rare DNA variant that causes failure of amplification and/or hybridization of a probe: for example, false homozygosity for p.Phe508del due to the presence of the variant F508C (c.1523T $>$ G); false homozygosity for p.lle507del due to the presence of p.lle507Val (I597V) (c.1519A>G).

- Uniparental disomy of chromosome 7, more rarely. 
Table 1 Examples of common CF-causing, indetermined, and non CF-causing variants (modified from ${ }^{5,8,17}$ )

\begin{tabular}{|c|c|c|}
\hline \multirow[b]{2}{*}{ Legacy name } & \multicolumn{2}{|c|}{ HGVS nomenclature } \\
\hline & cDNA nucleotide name & Protein name \\
\hline \multicolumn{3}{|c|}{ CF-causing variants ${ }^{\mathrm{a}}$} \\
\hline F508del & c.1521_1523deICTT & p.Phe508del \\
\hline G542X & c. $1624 G>T$ & p.Gly $542^{*}$ \\
\hline G551D & c. $1652 G>A$ & p.Gly551Asp \\
\hline N1303K & c. $3909 C>G$ & p.Asn 1303Lys \\
\hline W1282X & c. $3846 \mathrm{G}>\mathrm{A}$ & p.Trp1282* \\
\hline $621+1 G>T$ & c. $489+1 G>T$ & \\
\hline CFTRdele2,3 & $\begin{array}{l}\text { c.54-5940_273 } \\
+10250 \text { del21080 }\end{array}$ & p.Ser18Argfs*16 \\
\hline E60X & c. $178 \mathrm{G}>\mathrm{T}$ & p.Glu60* \\
\hline G85E & c. $254 \mathrm{G}>\mathrm{A}$ & p.Gly85Glu \\
\hline 394delTT & c.262_263deITT & p.Leu88Ilefs*22 \\
\hline $711+1 G>T$ & c. $579+1 G>T$ & \\
\hline R347P & c. $1040 G>C$ & p.Arg347Pro \\
\hline A455E & c. $1364 \mathrm{C}>\mathrm{A}$ & p.Ala455Glu \\
\hline Q493X & c. $1477 \mathrm{C}>\mathrm{T}$ & p.Gln493* \\
\hline 1507del & C.1519_1521delATC & p.lle507del \\
\hline R553X & c. $1657 \mathrm{C}>\mathrm{T}$ & p.Arg553* \\
\hline R560T & c. $1679 G>C$ & p.Arg560Thr \\
\hline $1898+1 G>A$ & c. $1766+1 G>A$ & \\
\hline $2183 A A>G$ & c.2051_2052delAAinsG & p.Lys684Serfs*38 \\
\hline $2789+5 G>A$ & c. $2657+5 G>A$ & \\
\hline $3120+1 G>A$ & c. $2988+1 G>A$ & \\
\hline M1101K & c. $3302 \mathrm{~T}>\mathrm{A}$ & p.Met1101Lys \\
\hline $\mathrm{R} 1162 \mathrm{X}$ & c. $3484 \mathrm{C}>\mathrm{T}$ & p.Arg1162* \\
\hline 3659deIC & c.3528delC & p.Lys 1177 Serfs* 15 \\
\hline M1V & c. $1 \mathrm{~A}>\mathrm{G}$ & p.? (unknown) \\
\hline Q39X & c. $115 \mathrm{C}>\mathrm{T}$ & p.Gln39* \\
\hline P67L & c. $200 \mathrm{C}>\mathrm{T}$ & p.Pro67Leu \\
\hline $\mathrm{R} 75 \mathrm{X}$ & c. $223 \mathrm{C}>\mathrm{T}$ & p.Arg75* \\
\hline $405+1 G>A$ & c. $273+1 G>A$ & \\
\hline $406-1 G>A$ & c. $274-1 G>A$ & \\
\hline E92X & c. $274 G>T$ & p.Glu92* \\
\hline E92K & c. $274 G>A$ & p.Glu92Lys \\
\hline Q98X & c. $292 C>T$ & p.Gln98* \\
\hline 457TAT $>$ G & c.325_327delTATinsG & p.Tyr 109Glyfs*4 \\
\hline $\mathrm{D} 110 \mathrm{H}$ & c. $328 \mathrm{G}>\mathrm{C}$ & p.Asp $110 \mathrm{His}$ \\
\hline $\mathrm{R} 117 \mathrm{C}$ & c. $349 \mathrm{C}>\mathrm{T}$ & p.Arg117Cys \\
\hline Y122X & c. $366 \mathrm{~T}>\mathrm{A}$ & p.Tyr $122^{*}$ \\
\hline 574delA & c. 442 delA & p.lle148Leufs*5 \\
\hline 444delA & c.313delA & p.lle105Serfs*2 \\
\hline 663delT & c.531delT & p.lle177Metfs*12 \\
\hline G178R & c. $532 \mathrm{G}>\mathrm{A}$ & p.Gly178Arg \\
\hline $711+3 A>G$ & c. $579+3 A>G$ & \\
\hline $711+5 G>A$ & c. $579+5 G>A$ & \\
\hline $712-1 G>T$ & c. $580-1 \mathrm{G}>\mathrm{T}$ & \\
\hline H199Y & c. $595 \mathrm{C}>\mathrm{T}$ & p.His199Tyr \\
\hline P205S & c. $613 \mathrm{C}>\mathrm{T}$ & p.Pro205Ser \\
\hline L206W & c. $617 \mathrm{~T}>\mathrm{G}$ & p.Leu206Trp \\
\hline Q220X & c. $658 \mathrm{C}>\mathrm{T}$ & p.G $\ln 220^{*}$ \\
\hline 852 del22 & $\begin{array}{l}\text { c.720_741delAGGGAGAAT } \\
\text { GATGATGAAGTAC }\end{array}$ & p.Gly241Glufs*13 \\
\hline 1078delT & c.948delT & p.Phe316Leufs*12 \\
\hline G330X & c. $988 \mathrm{G}>\mathrm{T}$ & p.Gly330* \\
\hline
\end{tabular}

Table 1 (Continued)

\begin{tabular}{|c|c|c|}
\hline \multirow[b]{2}{*}{ Legacy name } & \multicolumn{2}{|c|}{ HGVS nomenclature } \\
\hline & cDNA nucleotide name & Protein name \\
\hline R334W & c. $1000 \mathrm{C}>\mathrm{T}$ & p.Arg334Trp \\
\hline I336K & c. $1007 \mathrm{~T}>\mathrm{A}$ & p.lle336Lys \\
\hline T338I & c. $1013 \mathrm{C}>\mathrm{T}$ & p.Thr338lle \\
\hline 1154insTC & c.1021_1022dupTC & p.Phe 342 Hisfs $* 28^{*}$ \\
\hline S341P & c. $1021 \mathrm{~T}>\mathrm{C}$ & p.Ser341Pro \\
\hline R347H & c. $1040 \mathrm{G}>\mathrm{A}$ & p.Arg347His \\
\hline 1213delT & c.1081delT & p.Trp361Glyfs*8 \\
\hline $1248+1 G>A$ & c. $1116+1 \mathrm{G}>\mathrm{A}$ & \\
\hline 1259insA & c.1130dupA & p.GIn378Alafs*4 \\
\hline W401X(TAG) & c. $1202 \mathrm{G}>\mathrm{A}$ & p. $\operatorname{Trp} 401^{*}$ \\
\hline W401X(TGA) & c. $1203 \mathrm{G}>\mathrm{A}$ & p.Trp401* \\
\hline $1341+1 G>A$ & c. $1209+1 G>A$ & \\
\hline 1461 ins 4 & c.1329_1330insAGAT & p.lle444Argfs $* 3$ \\
\hline $1525-1 G>A$ & c. $1393-1 G>A$ & \\
\hline S466X & c. $1397 C>A$ or $c .1397 C>G$ & p.Ser466* \\
\hline L467P & c. $1400 \mathrm{~T}>\mathrm{C}$ & p.Leu467Pro \\
\hline S489x & c. $1466 \mathrm{C}>\mathrm{A}$ & p.Ser489* \\
\hline S492F & c. $1475 \mathrm{C}>\mathrm{T}$ & p.Ser492Phe \\
\hline 1677delTA & c.1545_1546delTA & p.Tyr515* \\
\hline V520F & c. $1558 \mathrm{G}>\mathrm{T}$ & p.Val520Phe \\
\hline $1717-1 G>A$ & c. $1585-1 G>A$ & \\
\hline $1717-8 G>A$ & c. $1585-8 G>A$ & \\
\hline S549R & c. $1645 \mathrm{~A}>\mathrm{C}$ & p.Ser549Arg \\
\hline S549N & c. $1646 \mathrm{G}>\mathrm{A}$ & p.Ser549Asn \\
\hline S549R & c. $1647 \mathrm{~T}>\mathrm{G}$ & p.Ser549Arg \\
\hline Q552X & c. $1654 \mathrm{C}>\mathrm{T}$ & p.Gln552* \\
\hline A559T & c. $1675 G>A$ & p.Ala559Thr \\
\hline $1811+1.6 \mathrm{kbA}>\mathrm{G}$ & c. $1680-886 A>G$ & \\
\hline $1812-1 G>A$ & c. $1680-1 \mathrm{G}>\mathrm{A}$ & \\
\hline R560K & c. $1679 \mathrm{G}>\mathrm{A}$ & p.Arg560Lys \\
\hline E585X & c. $1753 \mathrm{G}>\mathrm{T}$ & p.Glu585* \\
\hline $1898+3 A>G$ & c. $1766+3 A>G$ & \\
\hline 2143delT & c.2012delT & p.Leu671* \\
\hline 2184insA & c.2052_2053insA & p.Gln685Thrfs*4 \\
\hline 2184delA & c.2052delA & p. Lys 684 Asnfs $^{*} 38$ \\
\hline R709X & c. $2125 \mathrm{C}>\mathrm{T}$ & p.Arg709* \\
\hline K710X & c. $2128 \mathrm{~A}>\mathrm{T}$ & p.Lys710* \\
\hline 2307insA & c.2175dupA & p.Glu726Argfs*4 \\
\hline L732X & c. $2195 \mathrm{~T}>\mathrm{G}$ & p.Leu732* \\
\hline 2347delG & c. $2215 \mathrm{delG}$ & p.Val739Tyrfs*16 \\
\hline R764X & c. $2290 \mathrm{C}>\mathrm{T}$ & p.Arg764* \\
\hline 2585delT & c.2453delT & p.Leu818Trpfs*3 \\
\hline E822X & c. $2464 G>T$ & p.Glu822* \\
\hline $2622+1 G>A$ & c. $2490+1 G>A$ & \\
\hline E831X & c. $2491 G>T$ & p.Glu831* \\
\hline W846X & c. $2537 G>A$ & p.Trp846* \\
\hline W846X & c. $2538 \mathrm{G}>\mathrm{A}$ & p.Trp846* \\
\hline (2670TGG > TGA) & & \\
\hline R851X & c. $2551 \mathrm{C}>\mathrm{T}$ & p.Arg851* \\
\hline 2711delT & c.2583delT & p.Phe861Leufs*3 \\
\hline S945L & c. $2834 \mathrm{C}>\mathrm{T}$ & p.Ser945Leu \\
\hline $2789+2$ ins A & c. $2657+2 \_2657+3$ ins $A$ & \\
\hline Q890X & c. $2668 \mathrm{C}>\mathrm{T}$ & p.GIn890* \\
\hline L927P & c. $2780 \mathrm{~T}>\mathrm{C}$ & p.Leu927Pro \\
\hline 3007delG & c. 2875 delG & p.Ala959Hisfs*9 \\
\hline G970R & c. $2908 \mathrm{G}>\mathrm{C}$ & p.Gly970Arg \\
\hline $3120 \mathrm{G}>\mathrm{A}$ & c. $2988 G>A$ & \\
\hline
\end{tabular}


Table 1 (Continued)

HGVS nomenclature

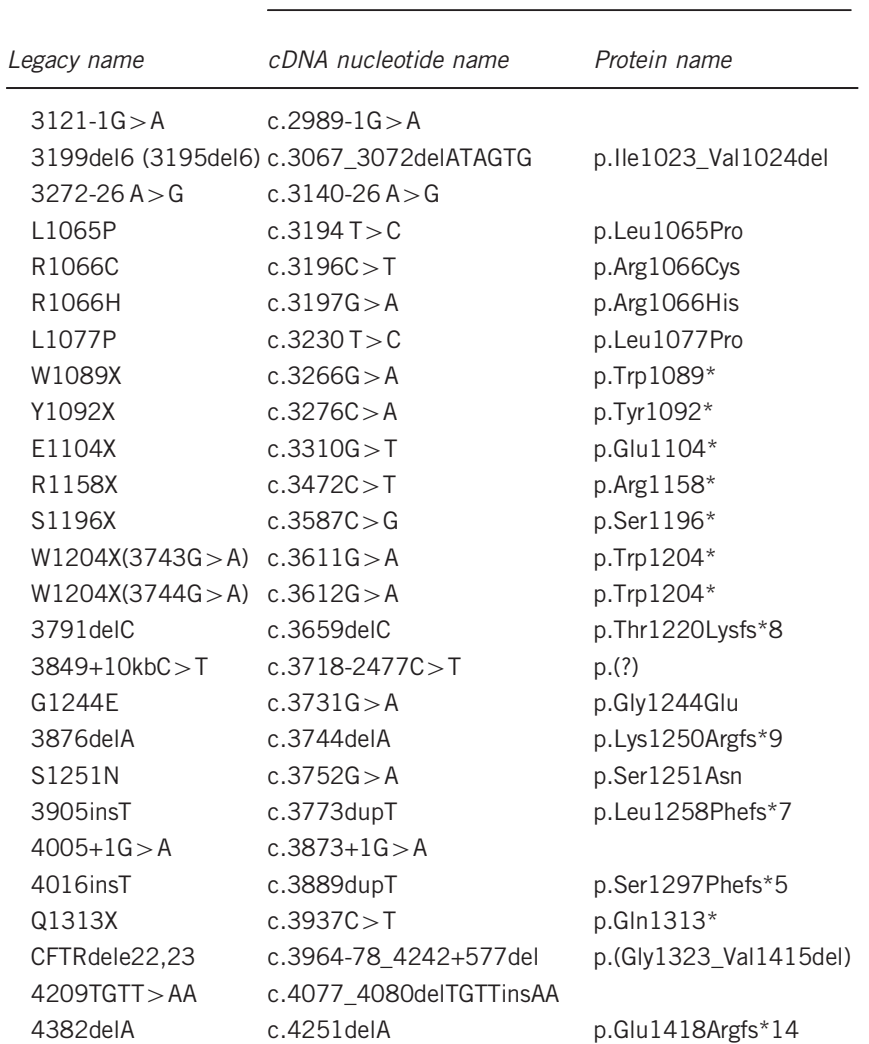

Examples of common variants with varying or indetermined clinical consequences ${ }^{\mathrm{b}}$

\begin{tabular}{|c|c|c|}
\hline $\mathrm{R} 117 \mathrm{H}$ & c. $350 G>A$ & p.Arg117His \\
\hline L227R & c. $680 \mathrm{~T}>\mathrm{G}$ & p.Leu227Arg \\
\hline 359K/T360K & c. $[1075 C>A ; 1079 C>A]$ & $\begin{array}{l}\text { p.[GIn359Lys; } \\
\text { Thr360Lys] }\end{array}$ \\
\hline L558S & c. $1673 \mathrm{~T}>\mathrm{C}$ & p.Leu558Ser \\
\hline Y569D & c. $1705 \mathrm{~T}>\mathrm{G}$ & p.Tyr569Asp \\
\hline D579G & c. $1736 A>G$ & p.Asp579Gly \\
\hline D614G & c. $1841 \mathrm{~A}>\mathrm{G}$ & p.Asp614Gly \\
\hline S977F & c. $2930 \mathrm{C}>\mathrm{T}$ & p.Ser977Phe \\
\hline F1052V & c. $3154 \mathrm{~T}>\mathrm{G}$ & p.Phe1052Val \\
\hline G1069R & c. $3205 \mathrm{G}>\mathrm{A}$ & p.Gly1069Arg \\
\hline $\mathrm{R} 1070 \mathrm{Q}$ & c. $3209 \mathrm{G}>\mathrm{A}$ & p.Arg1070GIn \\
\hline $\mathrm{D} 1152 \mathrm{H}$ & c. $3454 \mathrm{G}>\mathrm{C}$ & p.Asp $1152 \mathrm{His}$ \\
\hline $234 \mathrm{~V}$ & c. $3700 A>G$ & p.lle1234Val \\
\hline
\end{tabular}

5T c. $1210-12[5]$

Examples of common not $\mathrm{CF}^{- \text {-causing variants }}{ }^{\mathrm{C}}$

\begin{tabular}{|c|c|c|}
\hline R31C & c. $91 \mathrm{C}>\mathrm{T}$ & p.Arg31Cys \\
\hline R74W & c. $220 \mathrm{C}>\mathrm{T}$ & p.Arg74Trp \\
\hline$R 75 Q$ & c. $224 \mathrm{G}>\mathrm{A}$ & p.Arg75GIn \\
\hline I148T & c. $443 \mathrm{~T}>\mathrm{C}$ & p.lle148Thr \\
\hline M470V & c. $1408 A>G$ & p.Met470Val \\
\hline G576A & c. $1727 \mathrm{G}>\mathrm{C}$ & p.Gly576Ala \\
\hline R668C & c. $2002 \mathrm{C}>\mathrm{T}$ & p.Arg668Cys \\
\hline V754M & c. $2260 \mathrm{G}>\mathrm{A}$ & p.Val754Met \\
\hline L997F & c. $2991 \mathrm{G}>\mathrm{C}$ & p.Leu997Phe \\
\hline I1027T & c. $3080 \mathrm{~T}>\mathrm{C}$ & p.lle1027Thr \\
\hline R1070W & c. $3208 C>T$ & p.Arg1070Trp \\
\hline R1162L & c. $3485 G>T$ & p.Arg1162Leu \\
\hline
\end{tabular}

Table 1 (Continued)

HGVS nomenclature

\begin{tabular}{lll} 
Legacy name & cDNA nucleotide name & Protein name \\
\hline S1235R & c.3705 T $>$ G & p.Ser1235Arg \\
D1270N & c.3808G $>$ A & p.Asp1270Asn \\
7T & c.1210-12[7] &
\end{tabular}

Abbreviation: HGVS, Human Genome Variation Society.

aCF-causing variants: when paired together in trans, they cause cystic fibrosis in most patients* The six most frequent non-p.Phe508del variants are listed first followed by 18 other common variants, then 110 other variants are listed by cDNA nucleotide numbering (middle column). CF-causing variants include: (i) variants that are predicted to introduce a premature termination codon (PTC) into CFTR mRNA and therefore result in absence of protein such as nonsense variants (sequence changes that introduce a stop codon), variants that affect canonical

nucleotides of the donor or acceptor splice sites (gt or ag) or insertions and deletions that cause a frameshift of the ORF (Open Reading Frame); (ii) variants occurring within or near splice sites that lead to severe reduction (>90\%) in the level of full-length CFTR mRNA and CFTR protein; (iii) missense variants that drastically alter folding, processing, trafficking and/or function of CFTR protein. *However, several variants in the list above may retain residual function and can be found either in CF or in CFTR-RDs. Well known examples include missense variants D110H, R117C, L206W, R347P, R347H, R1066H, or splice variants that produce both aberrant and full-length transcript such as $3849+10 k b C>T, 2789+5 G>A, 3272-26 A>G, 711+3 A>G$.

VVariants with varying or indetermined clinical consequences: when paired with a severe variant in trans, they cause either no disease phenotype, or CFTR-RD or mild CF-PS. The 5 T allele is the most common allele associated with CFTR-RDs worldwide. The disease penetrance of some of these variants is dependent on other factors, such as other sequence changes associated in cis. The most studied examples are $\mathrm{R} 117 \mathrm{H}$ and the $5 \mathrm{~T}$ allele. When paired in trans with a severe $\mathrm{CF}$ variant, $\mathrm{R} 117 \mathrm{H}$ is considered as causing CFTR-RD (or rarely mild CF-PS) if associated in cis with a 5T allele (rare occurence), whereas it is considered as a neutral or CFTR-RD variant when associated in cis with the common $7 \mathrm{~T}$ allele. ${ }^{11}$ Owing to the extremely low penetrance of $\mathrm{R} 117 \mathrm{H}$ for $\mathrm{CF}^{18}$ in some countries this variant has been removed from the newborn CF screening panels, as its presence created both management and counseling dilemmas (many healthy carriers of R117H-7 T could be wrongly considered as CF carriers and prenatal diagnosis inappropriately proposed).

'Non CF-causing variants: when paired with a severe variant in trans, they cause usually no disease phenotype, or a CFTR-RD in some individuals. Missense variants R74W, R1070W, D1270N are classified as 'indeterminate' by Sosnay et al., ${ }^{17}$ however, as they are frequently found in trans with a severe CF variant in asymptomatic individuals (including fertile fathers), they may not be sufficient to cause disease. 19 Moreover, they are often associated within the same allele (eg in cis), forming various combinations ('complex alleles') depending on individuals, so that their disease liability is questionable. individuals,

R74W;D1270N] C.[220C $>T ; 3808 G>A]$

[R74W;V201M;D1270N] C.[220C $>T ; 601 G>A ; 3808 G>A]$

[R74W:R1070W;D1270N] C. [220C > T;C.3208C > T:3808G >A]

$1148 \mathrm{~T}$ is a neutral variant, but can be associated in cis with a severe CF variant

c.3067 3072del (legacy 3199del6 or 3195del6) that, in isolation causes CF, whereas I148T

in isolation does not. ${ }^{19,20} \mathrm{G} 576 \mathrm{~A}$ is found in cis with R668C and R668C can be found alone or in cis with G576A. I1027T is usually found in cis with F508del:

Notes: (i) Some missense variants classified as either indeterminate or non CF-causing (R74Q, R75Q, R117H, R170H, L967S, L997F, D1152H, S1235R and D1270N) can selectively alter the bicarbonate permeation of the CFTR channel (but not the chloride channel), thus affecting primarily the organs that utilize CFTR for bicarbonate secretion (pancreas, nasal sinus, or vas deferens) and consequently, they could be involved in the pathogenic mechanisms of CFTRdeferens) and, consequently, they could be involved in the pathogenic mechanisms of CFTRthe HGVS version in order to ensure compatibility with clinical reports and understanding by clinicians and couples. In the associated text, for reasons of space, some CFTR variants may be referred using traditional names.

function variants that cause CF disease when paired together; (ii) variants that retain residual CFTR function and are compatible with milder phenotypes such as CFTR-RD; (iii) variants with no clinical consequences; and (iv) variants of unproven or uncertain clinical relevance. A recent survey of genotype and phenotype data from almost 40000 individuals recorded as 'affected with CF' in the clinical registries in Europe and North America, in conjunction with functional assessment, allowed to redefine the disease liability of 159 common variants in the CFTR gene. ${ }^{17}$ However, the disease penetrance of the vast majority of hundreds of rare missense variants identified so far remains unknown. It is therefore recommended to work in close collaboration with specialized clinical geneticists and reference laboratories that have gained considerable knowledge in the interpretation of CFTR variants.

Otherwise, with respect to variations in local or national regulations and specific laboratory practices, the Montpellier meeting revealed some discrepancies in the way that couples are included or not in CF-PGD programs (Table 4). In several countries, when at least one 
Table 2 Examples of CFTR genotypes in traditional (legacy) and HGVS nomenclatures (modified and updated from Berwouts et al., 2011) ${ }^{41}$

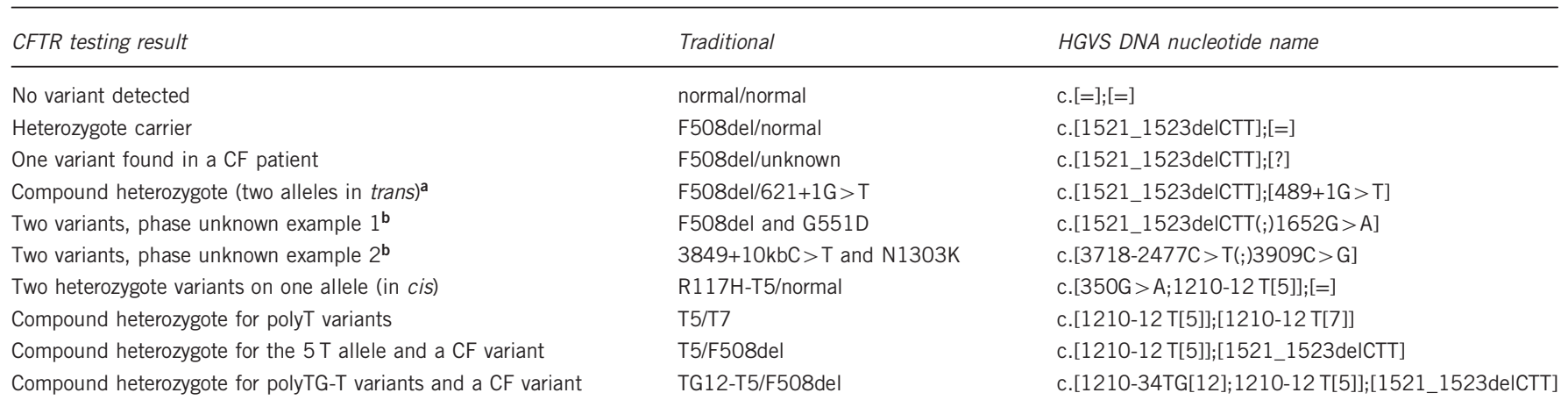

'c.' indicates a coding DNA sequence. In the HGVS nomenclature, variations are described at the nucleotide level because the use of a protein-based nomenclature may be equivocal. Example: p.Phe508del can be caused by different sequence changes at the DNA level, including c.1521_1523delCTT and c.1522 1524delTTT. See the HGVS nomenclature website (http://www.hgvs.org/ mutnomen/recs.html) for detailed descriptions of sequence variants and significance of punctuation symbols.

aThe term 'compound heterozygote' means that the phase of variants has been checked by testing both parents, which confirmed that each of them is a carrier of one of the two variants, which consequently are in trans (on opposite chromosomes) in the affected individual.

bThe phase of the two variants has not been checked by parental testing: compound heterozygosity cannot be confirmed.

\section{Box 3 Internet resources}

\begin{tabular}{ll}
\hline CF-Network EQA_External Quality Assessment process & http://www.cfnetwork.be \\
United Kingdom National External Quality Assessment Service for Molecular Genetics (UKNEQAS) & http://www.ukneqas-molgen.org.uk \\
EuroGentest & http://www.eurogentest.org/web/qa/basic/xhtml \\
HGVS nomenclature & http://www.hgvs.org/mutnomen/ \\
European Molecular Genetics Quality Network (EMQN) & http://www.emqn.org/emqn/Best+Practice \\
Cystic Fibrosis Mutation Database & http://www.genet.sickkids.on.ca/ \\
CFTR2 & http://www.cftr2.org \\
Testing guidelines for molecular diagnosis of cystic fibrosis (2008) & www.acgs.uk.com/media/774379/cfbp26may.pdf \\
Orphanet & www.orpha.net \\
OCDE guidelines for quality assurance in molecular genetic testing & http://www.oecd.org/sti/biotech/38839788.pdf \\
University of California Santa Cruz (UCSC) genome browser & http://genome.ucsc.edu
\end{tabular}

\section{Table 3 Main indications for CFTR testing}

Newborn screening for $\mathrm{CF}$

Diagnostic testing

Typical CF clinical presentations (including newborns with meconium ileus) Atypical clinical presentations and/or borderline or negative sweat test

Male infertility with CBAVD

CFTR-Related Disorder in adults

Fetuses with bowel hyperechogenicity and/or loop dilatation in the second trimester of pregnancy (risk of CF estimated as 3\%)

\section{Carrier testing}

Individuals with a family history of CF ('cascade testing')

Individuals without a family history (essentially for preconception carrier screening)

Partner of a CF patient

Partner of a CBAVD patient

Infertile couples seeking for IVF (not usually recommended among European genetic societies, as the risk of carrying a CF variant is not significantly higher than that of the general population).

Prenatal diagnosis, Preimplantation genetic diagnosis, offered to

Parents of a CF patient

Carrier couples identified through carrier testing

Carrier couples identified through investigations for fetal bowel anomalies

Couples with one individual affected with CF and a carrier partner

Couples with one individual affected with CBAVD and a carrier partner
Table 4 Inclusion and exclusion criteria for CF-PGD according to countries

\begin{tabular}{lccccccc}
\hline & France & Italy & Spain & Belgium & Greece & UK & USA \\
\hline Parents & & & & & & & \\
1 affected+1 carrier S/LS & A & A & A & A & A & A & A \\
1 affected+1 carrier M & R & A & A & A & A & A & A \\
1 affected+1 carrier U & A & A & A & A & R & A & A \\
2 carriers S/LS & A & A & A & A & A & A & A \\
1 carrier S/LS+1 carrier M & R & A & A & A & A & A & A \\
1 carrier S/LS+1 carrier U & A & A & A & A & R & A & A \\
\hline
\end{tabular}

Abbreviations: S, severe CF-causing variant (p.Phe508del, p.Gly542^...); LS, large spectrum variant (p.Leu206Trp, c. $2657+5 G>A$...); M, mild variant with variable disease penetrance (IVS8-5 T allele, p.Arg117His-7 T ...); U, variants of unproven or uncertain CF clinical relevance (p.Pro1013His, p.Arg1162Leu ...); A, accepted; R, refused.

$\star$ In the context of a family history of Cystic Fibrosis (see text). Population carrier screening is not performed in the UK.

severe CF-causing or large spectrum variant is identified in one of the members of the couple, PGD is accepted even though a mild or benign variant is found in the other partner. This is an exclusion criterion in France where PGD is restricted to couples 'having a high probability to give birth to a child affected by a particularly severe genetic disease and recognized as being incurable at the time of diagnosis'. Consequently, only the risk of transmitting CF (and not milder phenotypes such as CFTR-RD) is considered for PGD. In the UK, when one couple member is heterozygous for a variant of unproven or uncertain clinical relevance, PGD is accepted only in the context of a 
Table 5 List of markers commonly used in PGD for Cystic Fibrosis

\begin{tabular}{|c|c|c|c|c|c|}
\hline Marker & Type & UniSTSa & Location on chromosome $7^{b}$ & Position according to the CFTR gene $e^{c}$ & Heterozygosity rate (\%) \\
\hline D7S677 & (ca)n & 18390 & $117,499,431 \_117,499,463$ & Intron 1 & 85 \\
\hline IVS10CA & (ca)n & NA & $117,566,250 \_117,566,288$ & Intron 11 & 89 \\
\hline IVS17BCA & (ca)n & NA & $117,612,236 \_117,612,262$ & Intron 20 & 40 \\
\hline IVS17BTA & $(\mathrm{ta}) \mathrm{n}$ & NA & $117,612,009 \_117,612,069$ & Intron 20 & 80 \\
\hline D7S2460 & (ca)n & 24290 & $116,767,976 \_116,768,008$ & Centromeric, $0.7 \mathrm{Mb}$ & 71 \\
\hline D7S23 & (ca)n & 31363 & $117,322,538 \_117,322,572$ & Centromeric, $0.18 \mathrm{Mb}$ & 85 \\
\hline D7S633 & (ca)n & 70412 & $117,370,804 \_117,370,850$ & Centromeric, $0.1 \mathrm{Mb}$ & 45 \\
\hline D7S2847 & (tatc)n & 64614 & $119,179,488 \_119,179,552$ & Telomeric, $1.46 \mathrm{Mb}$ & 84 \\
\hline AFM320vb5 & (ca)n & 31676 & $118,351,454 \_118,351,480$ & Telomeric, $0.64 \mathrm{Mb}$ & 51 \\
\hline
\end{tabular}

Abbreviation: NA, not applicable.

aUniSTS has migrated to UCSC

bAccording to UCSC GRCh38/hg38, December 2013.

'Exon numbering according to the HGVS nomenclature. Physical distances are taken from location along chromosome 7 according to the UCSC genome browser.

family history of CF. As a precaution, in all participating centres, novel rare variants of unproven or uncertain clinical significance may be considered as potentially CF-causing upon advice of specialists of CFTR alterations.

\section{GENETIC COUNSELING AND EMBRYO TRANSFER}

Similarly, discrepancies in the regulations about embryo transfer may exist from one country to another. It is recommended that all couples are individually counseled before the clinical cycle and the expected embryo genotypes and corresponding phenotypes are clearly explained.

(i) Rare variants or variants with incomplete penetrance in disease

Couples should be aware that in case of a genotype that includes rare variants with incomplete penetrance, individual outcomes cannot be reliably predicted. For instance, an embryo with p.Phe508del in trans with the 5T allele (genotype c.[1210-12 T[5]]; [1521_1523delCTT]) may be considered as suitable for transfer in some countries, while in others the parents' wish to preferentially transfer carrier embryos without the IVS8-5 T allele is taken into account. In countries in which embryos compound heterozygous for a severe and a mild/benign variant may be transferred, couples should be informed of possible risks for their children, ranging from no clinical signs to moderate and/or late-onset phenotypes. Some common or rare CFTR variants are associated with a wide phenotypic spectrum and updated information should be provided in light of current knowledge (Table 1).

(ii) Selection of embryos for transfer

Couples should be informed that following the identification of genetically transferable embryos a subsequent selection is made based upon the morphology of the embryo and its potential to implant. Thus morphology rather than distinction between carrier embryos and embryos free of both parental germ-line variants, forms the basis of the final selection of the embryo for transfer. Some centres may also include aneuploidy screening in the PGD protocol in order to achieve higher implantation and live birth rates. In these cases, couples need to be appropriately counseled as the identification of aneuploidy may exclude embryos that had been considered transferable on the basis of CFTR analysis alone. Couples should also be made aware that diagnosis may not be achieved for some embryos for various reasons including a failure of amplification or due to cumulus cell contamination. It is useful for the IVF unit to know the live birth rate per cycle started for CF-PGD at their center as well as the outcome following transfer after cryopreservation of surplus transferable embryos. Multiplex PCR protocols have proven to be robust, sensitive and highly accurate, ${ }^{12,31}$ however it is recommended in most countries that the results should be confirmed with a prenatal diagnostic test.

\section{PGD STRATEGY}

Recommended strategies and decision procedures for PGD are presented in the following section. In particularly difficult situations, it is essential that the diagnostic strategies be shared with the referring clinical geneticist.

As mentioned in the amplification-based PGD guidelines, ${ }^{26,27}$ it is recommended to perform both indirect (marker based) and direct (variant based) genetic diagnosis.

\section{PGD diagnostic strategies based on polymorphic markers}

Several microsatellite markers within or close to the CFTR gene can be used in PGD for CF (Table 5). Laboratories may have to search databases for previously unpublished microsatellite markers in order to obtain sufficient informative markers for a given couple.

It is recommended to offer PGD using at least two fully informative flanking markers within a 1-megabase $(\mathrm{Mb}$, equivalent to one million base pairs) region on both sides of the gene and to include intragenic markers when available.

As recombination events within the CFTR gene have been very rarely described, it is acceptable to perform indirect genetic diagnosis using intragenic microsatellites located on the same side of the variant if markers flanking the CFTR gene are not informative or gave inconclusive results.

When MDA is performed, it is recommended to study at least four fully informative microsatellite markers (two each side of the gene) because of the higher Allele Drop Out (ADO) rates frequently reported. Furthermore, semi-informative or noninformative markers can still be used in an informative haplotype to detect the presence of contamination and/or chromosomal abnormality (presence of one or three haplotypes).

All customized protocols must be thoroughly validated before clinical use. ${ }^{25,26}$ 


\section{PGD strategies based on variants identified in couples}

Case 1: p.Phe508del in one or both couple members. As the most frequent CF variant is the p.Phe508del in exon 11 of the CFTR gene (exon 10 in the traditional nomenclature), it is very common to identify this variant in at least one of the couple members (Box 4). Therefore, it is highly recommended to set up single-cell PCR conditions for the p.Phe508del variant in combination with indirect genetic diagnosis to monitor contamination and preclude misdiagnosis owing to $\mathrm{ADO}$.

For couples in whom one of the partners is heterozygous for a variant other than p.Phe508del, it is acceptable to perform a direct diagnosis for the p.Phe508del and an indirect diagnosis for the other variant, provided sufficient informative markers are available to trace inheritance of both chromosomes from the non-p.Phe508del carrier.

When there is a lack of informative markers in the parent carrier of the other variant, it is recommended to specifically study this causative variant (in addition to the p.Phe508del) and also to biopsy and independently analyze two cells instead of one, preferably at the blastocyst stage.

Case 2: Identical variant (other than p.Phe508del) in both couple members. It is recommended to develop PCR conditions for the unique CF-causing variant in addition to indirect genetic diagnosis. It is acceptable to perform only indirect genetic diagnosis for fully informative couples (at least two informative markers $<1 \mathrm{Mb}$ flanking the gene or one intragenic marker and one flanking marker).

Case 3: Different variants (other than p.Phe508del) in both couple members. It is acceptable to perform only indirect genetic diagnosis for fully informative couples (at least two informative markers $<1 \mathrm{Mb}$ flanking the gene or one intragenic marker and one flanking marker). In cases that lack informativity for markers within or near the gene, it is recommended to specifically study both causative variants and to search and add informative markers located further up- or downstream. The biopsy and analysis of trophectoderm cells at the blastocyst stage, should also help in reducing the misdiagnosis risk due to $\mathrm{ADO}$.

\section{REPORTING}

There is currently no formal consensus regarding PGD reports. It also depends on legislation and ethical laws of each country. However, according to recommendations for reporting results of diagnostic genetic testing, ${ }^{44}$ guidelines for accreditation of the PGD laboratory, ${ }^{45}$ and recommendations from OECD (Organization for Economic Co-operation and Development), some important items should be included in a PGD report. They are listed below.

\section{Box 4 Genotype coverage in CF}

With a frequency of p.Phe508del reaching $~ 70 \%$ of CF alleles in populations from Northern European descent, almost half of individuals with CF have two copies of the $p$. Phe $508 \mathrm{del}$ allele $\left(p^{2}=(0.7)^{2}=0.49\right), 42 \%$ have one $p$. Phe $508 \mathrm{del}$ in trans with another CFTR variant $(2 p q=2 \times 0.7 \times 0.3=0.42)$ and $9 \%$ have two non-p.Phe508del variants $\left(q^{2}=(0.3)^{2}=0.09\right)$. Commercially available testing panels including the 30 most common CF variants detect about $87 \%$ of $\mathrm{CF}$ alleles, so that it can be predicted that $24 \%$ of patients with CF carry at least one rare variant not included in these panels $\left(2 \times 0.87 \times 0.13+(0.13)^{2}=0.24\right)$. However, these features can be very different in Southern-European and nonEuropean populations, who exhibit a much larger proportion of rare variants and a decreased frequency of p.Phe508del.

\section{Feasibility report}

- Laboratory issuing results (affiliation, address, secretarial phone and fax numbers)

- Date of report

- Name of the clinician referring the couple

- Unequivocal identification of the couple (2 identifiers per individual, e.g. name and date of birth)

- Specimen information - type of sample and date of sampling (also time if appropriate)

- Disease (OMIM number, inheritance mode) and gene (OMIM number) being tested for

- Methods performed and markers used

- Clearly presented results with appropriate interpretative comments

- Appropriate reference intervals

- Error rates clearly stated

- Variant nomenclature using Human Genome Variation Society (HGVS) recommendations

- Accession number of reference sequence (including version number)

- Identification of individual providing results and authorizer

\section{PGD case report}

- Laboratory issuing results (affiliation, address, secretarial phone and fax numbers)

- Referring clinician: name and affiliation

- Date of report

- Title, that is 'PGD for cystic fibrosis'

- Disease (OMIM number and inheritance mode) and gene (OMIM number) being tested for

- Unequivocal identification of both couple members (2 identifiers per individual, e.g. name and date of birth)

- Specimen information: type of sample, date/time of sampling, date of arrival of samples in the lab

- Methods performed

- List of DNA sequences studied

- Names of the variants analyzed (using HGVS nomenclature)

Accession number of reference sequence (including version number)

- Limits of the test (risk of recombination, ADO rate if indicated)

- Results obtained (genotype may be given or not depending on local rules). Clearly presented results with appropriate interpretative comments with clear indication of whether embryos should or should not be transferred

- Error rates clearly stated

- Identification of individual providing results and authorizer

- The report must be signed (some labs sign all steps: experiments, reading, validation)

- Pagination to include the actual and total number of pages

The reports must be sent (faxed or emailed, depending on legislation on confidentiality of medical information) to the referring physician and/or IVF team as described in the official test request form, in time for embryo transfer. Results should be reported only to persons authorized to receive and use this kind of medical information. In some jurisdictions, the lab report must also be sent to the patient (ie Italy). 


\section{EXTERNAL QUALITY ASSESSMENT}

To meet the ISO 15189 standards, accredited laboratories are required to participate in EQA schemes, which provide an independent evaluation of the quality of their service and inter-laboratory comparison, with an educational purpose. The United Kingdom National External Quality Assessment Service has developed a scheme specifically for molecular PGD, based on experience gained with a three-years pilot scheme for CF-PGD. ${ }^{46}$ The scheme examines (i) the ability of each participating lab to genotype genomic DNA and single cells (ii) the interpretation of results (including the determination of the limitations of the protocol that was applied for the testing of single cell samples supplied in the EQA), and (iii) the reporting of PGD results. The scheme recommendations were followed by the participating laboratories and led to the improvement in the content of the reports.

\section{CONCLUDING REMARKS}

This meeting organized on behalf of EuroGentest has highlighted important variation among the participating countries both in technical protocols and in the counseling of couples. It emphasized the need to pursue harmonization and quality assurance, preferably through some form of internationally or nationally recognized accreditation. Given the complexity of phenotype and genotype relationships, the extremely high number of variants found in the CFTR gene, and the reclassification of some variants as (non)pathogenic as more data become available, genetic counseling is mandatory, and less common variants should be reviewed with CFTR experts before acceptance of a PGD CF request.

\section{CONFLICT OF INTEREST}

The authors declare no conflict of interest.

\section{ACKNOWLEDGEMENTS}

We acknowledge EuroGentest for funding and all participants of this meeting for fruitful discussions. EuroGentest was funded by the European Commission's Seventh Framework Program (contract HEALTH-F4-2010261469 for the EuroGentest2 project). We express our sincere gratitude to Professor Gert Matthijs for providing corrections, suggestions and encouragement.

1 Salvatore D, Buzzetti R, Baldo E et al: An overview of international literature from cystic fibrosis registries. Part 3. Disease incidence, genotype/phenotype correlation, microbiology, pregnancy, clinical complications, lung transplantation, and miscellanea. J Cyst Fibros 2011; 10: 71-85.

2 Castellani C, Southern KW, Brownlee $\mathrm{K}$ et al: European best practice guidelines for cystic fibrosis neonatal screening. J Cyst Fibros 2009; 8: 153-173.

3 Massie RJ, Curnow L, Glazner J, Armstrong DS, Francis I: Lessons learned from 20 years of newborn screening for cystic fibrosis. Med J Aust 2012; 196: 67-70.

4 Wagener JS, Zemanick ET, Sontag MK: Newborn screening for cystic fibrosis. Curr Opin Pediatr 2012; 24: 329-335.

5 Bombieri C, Claustres M, De Boeck K et al: Recommendations for the classification of CFTR-related disorders. J Cyst Fibros 2011; 10: S86-102.

6 Dumur V, Gervais R, Rigot JM et al: Abnormal distribution of CF deltaF508 allele in azoospermic men with congenital aplasia of epididymis and vas deferens. Lancet 1990; 336: 512

7 Chillon M, Casals T, Mercier B et al: Mutations in the cystic fibrosis gene in patients with congenital absence of the vas deferens. New Engl J of Med 1995; 332: 147-1480.

8 Claustres M, Guittard C, Bozon D et al: Spectrum of CFTR mutations in cystic fibrosis and in congenital absence of the vas deferens in France. Hum Mutat 2000; 16: 143-156.

9 Groman JD, Hefferon TW, Casals T et al: Variation in a repeat sequence determines whether a common variant of the cystic fibrosis transmembrane conductance regulator gene is pathogenic or benign. Am J Hum Genet 2004; 74: 176-179.
10 Claustres M: Molecular pathology of the CFTR locus in male fertility. Reprod Biomed Online 2005; 10: 14-41.

11 Thauvin-Robinet C, Munck A, Huet F et al: CFTR p.Arg117H is associated with CBAVD and other CFTR-related disorders. J Med Genet 2013; 50: 220-227.

12 Girardet A, Ishmukhametova A, Willems $M$ et al: Preimplantation genetic diagnosis for cystic fibrosis: the Montpellier center's 10-year experience. Clin Genet 2015; 87: 124-132.

13 Masson E, Chen J-M, Audrezet MP, Cooper DN, Ferec C: A conservative assessment of the major genetic causes of idiopathic chronic pancreatitis: data from a comprehensive analysis of PRSS1, SPINK1, CTRC and CFTR genes in 253 young French patients. Plos One 2013; 8: e73522.

14 LaRusch J, Jung J, General IJ et al: Mechanisms of CFTR functional variants that impair regulated bicarbonate permeation and increase risk for pancreatitis but not for cystic fibrosis. PLoS Genet 2014; 10: e1004376.

15 Girodon E, Cazeneuve C, Lebargy F et al: CFTR gene mutations in adults with disseminated bronchiectasis. Eur J Hum Genet 1997; 5: 149-155.

16 Cystic Fibrosis Foundation, Borowitz D, Parad RB et al: Cystic Fibrosis Foundation practice guidelines for the management of infants with cystic fibrosis transmembrane conductance regulator-related metabolic syndrome during the first two years of life and beyond. J Pediatr 2009; 155: S106-S116.

17 Sosnay PR, Siklosi KR, Van Goor F et al: Defining the disease liability of variants in the cystic fibrosis transmembrane conductance regulator gene. Nat Genet 2013; 45 1160-1167.

18 Thauvin-Robinet C, Munck A, Huet F et al: The very low penetrance of cystic fibrosis for the $\mathrm{R} 117 \mathrm{H}$ mutation: a reappraisal for genetic counselling and newborn screening. J Med Genet 2009; 46: 752-758.

19 Claustres M, Altieri JP, Guittard C, Templin C, Chevalier-Ports F, Des Georges M: Are p.148 T, p.R74W and p.D1270N CF causing mutations? BMC Med Genet 2004; 5: 19.

20 Monaghan KG, Highsmith WE, Amos J et al: Genotype-phenotype correlation and frequency of the 3199del 6 cystic fibrosis mutation among I148T carriers: results from a collaborative study. Genet Med 2004; 6: 421-425.

21 Raynal C, Baux D, Theze $C$ et al: A classification model relative to splicing for variants of unknown clinical significance: application to the CFTR gene. Hum Mutat 2013; 34: 774-784.

22 Handyside AH, Kontogianni EH, Hardy K, Winston RM: Pregnancies from biopsied human preimplantation embryos sexed by Y-specific DNA amplification. Nature 1990; 344: 768-770.

23 Scott RT, Upham KM, Forman EJ, Zhao T, Treff NR: Cleavage-stage biopsy significantly impairs human embryonic implantation potential while blastocyst biopsy does not: a randomized and paired clinical trial. Fertil Steril 2013; 100: 624-630.

24 Retchisky S, Pakhalchuk T, San Ramos G, Goodman A, Zlatopolsky Z, Kuliev A: First systematic experience of preimplantation genetic diagnosis for single-gene disorders, and/or preimplantation human leukocyte antigen typing, combined with 24-chromosome aneuploidy testing. Fertil Steril 2015; 103: 503-512.

25 Moutou C, Goossens V, Coonen E et al: ESHRE PGD consortium data collection XII: cycles from January to December 2009 with pregnancy follow-up to October 2010. Hum Reprod 2014; 29: 880-903.

26 Thornhill AR, de Die-Smulders CE, Geraedts JP et al: ESHRE PGD Consortium "best practice guidelines for clinical preimplantation genetic diagnosis (PGD) and preimplantation genetic screening (PGS)". Hum Reprod 2005; 20: 35-48.

27 Harton GL, De Rycke M, Fiorentino F et al: ESHRE PGD consortium best practice guidelines for amplification-based PGD. Hum Reprod 2011; 26: 33-40.

28 Ray P, Ao A, Taylor D, Winston R, Handyside A: Assessment of the reliability of single blastomere analysis for preimplantation diagnosis of the $\Delta \mathrm{F} 508$ deletion causing cystic fibrosis in clinical practice. Prenat Diagn 1998; 18: 1402-1412.

29 Goossens V, Sermon K, Lissens W et al: Clinical application of preimplantation genetic diagnosis for cystic fibrosis. Prenat Diagn 2000; 20: 571-581.

30 Dreesen J, Jacobs L, Bras M et al: Multiplex PCR of polymorphic markers flanking the CFTR gene; a general approach for preimplantation genetic diagnosis of cystic fibrosis. Mol Hum Reprod 2000; 6: 391-396.

31 Moutou C, Gardes N, Viville S: Duplex, triplex and quadruplex PCR for the preimplantation genetic diagnosis (PGD) of cystic fibrosis (CF), an exhaustive approach. Prenat Diagn 2004; 24: 562-569.

32 Fiorentino F, Magli M, Podini D et al: The minisequencing method, an alternative strategy for preimplantation genetic diagnosis of single gene disorders. Mol Hum Reprod 2003; 7: 399-410.

33 Keymolen K, Goossens V, De Rycke M et al: Clinical outcome of preimplantation genetic diagnosis for cystic fibrosis: the Brussels'experience. Eur J Hum Genet 2007; 15: 752-758.

34 Renwick P, Trussler J, Lashwood A, Braude P, Mackie Ogilvie C: Preimplantation genetic haplotyping: 127 diagnostic cycles demonstrating a robust, efficient alternative to direct mutation testing on single cells. Reprod Biomed Online 2010; 20: 470-476.

35 Hellani A, Coskun S, Tbakhi A, Al-Hassan S: Clinical application of multiple displacement amplification in preimplantation genetic diagnosis. Reprod Biomed Online 2005; 10: 376-380.

36 Darcy D, Tian L, Taylor J, Schrijver I: Cystic fibrosis carrier screening in obstetric clinical practice: knowledge, practices, and barriers, a decade after publication of screening guidelines. Genet Test Mol Biomarkers 2011; 15: 517-523.

37 Harton G, Braude P, Lashwood A, Schmutzler A, Traeger-Synodinos J, Wilton L, Harper JC., ESHRE PGD: consortium best practice guidelines for organization of a PGD centre for PGD/preimplantation genetic screening. Hum Reprod 2011; 26: 14-24. 
38 Harton GL, Magli MC, Lundin K, Montag M, Lemmen J, Harper JC, ESHRE PGD: Consortium/Embryology Special Interest Group - best practice guidelines for polar body and embryo biopsy for preimplantation genetic diagnosis/screening (PGD/PGS). Hum Reprod 2011; 26: 41-46.

39 Beutler E, McKusick VA, Motulsky AG, Scriver CR, Hutchinson F: Mutation nomenclature: nicknames, systematic names, and unique identifiers. Hum Mutat 1996; 8: 203-206.

40 Den Dunnen JT, Antonarakis SE: Mutation nomenclature extensions and suggestions to describe complex mutations: a discussion. Hum Mutat 2000; 15: 7-12.

41 Berwouts S, Morris MA, Girodon E, Schwarz M, Stuhrmann M, Dequeker E: Mutation nomenclature in practice: findings and recommendations from the cystic fibrosis external quality assessment scheme. Hum Mutat 2011; 32: 1197-1203.

42 Strom CM, Redman JB, Peng M: The dangers of including nonclassical cystic fibrosis variants in population-based screening panels: p.L997F, further genotype/phenotype correlation data. Genet Med 2011; 13: 1042-1044.

43 Thomas M, Lemonnier L, Gulmans V et al: Is there evidence of correct diagnosis in cystic fibrosis registries? J Cyst Fibros 2014; 13: 275-280.

44 Claustres M, Kožich V, Dequeker E et al: Recommendations for reporting results of diagnostic genetic testing (biochemical, cytogenetic and molecular genetic). Eur J Hum Genet 2014; 22: 160-170.
45 Harper JC, SenGupta S, Vesela K et al: Accreditation of the PGD laboratory. Hum Reprod 2010; 25: 1051-1065.

46 Deans Z, Fiorentino F, Biricik A et al: The experience of 3 years of external quality assessment of preimplantation genetic diagnosis for cystic fibrosis. Eur J Hum Genet 2013; 21: 800-806.

cc) (i) $(\Theta)$ This work is licensed under a Creative Commons Attribution-NonCommercial-NoDerivs 4.0 International License. The images or other third party material in this article are included in the article's Creative Commons license, unless indicated otherwise in the credit line; if the material is not included under the Creative Commons license, users will need to obtain permission from the license holder to reproduce the material. To view a copy of this license, visit http://creativecommons.org/licenses/ by-nc-nd/4.0/ 